\title{
Infectability of Human BrainSphere Neurons Suggests Neurotropism of SARS-CoV-2
}

\author{
C. Korin Bullen" $\# 1$, Helena T. Hogberg\#2, Asli Bahadirli-Talbott1, William R. Bishai1, Thomas Hartung2,3,4, \\ Casey Keuthan 5, Monika M. Looney1, Andrew Pekosz4, J. Carolina Romero2, Fenna C. M. Sillé2,6, Peter Um ${ }^{1}$ \\ and Lena Smirnova2,\# \\ ${ }^{1}$ Johns Hopkins University, School of Medicine, Department of Medicine, Division of Infectious Diseases, Baltimore, MD, USA; ${ }^{2}$ Johns Hopkins \\ University, Bloomberg School of Public Health, Center for Alternatives to Animal Testing (CAAT), Baltimore, MD, USA; ${ }^{3}$ CAAT-Europe, University of \\ Konstanz, Konstanz, Germany; ${ }^{4}$ Johns Hopkins University, Bloomberg School of Public Health, Department of Molecular Microbiology and Immunology, \\ Baltimore, MD, USA; ${ }^{5}$ Johns Hopkins University, School of Medicine, Department of Ophthalmology, Wilmer Eye Institute, Baltimore, MD, USA; \\ ${ }^{6}$ Johns Hopkins University, Bloomberg School of Public Health, Department of Environmental Health \& Engineering, Baltimore, MD, USA
}

\begin{abstract}
Reports from Wuhan suggest that 36\% of COVID-19 patients show neurological symptoms, and cases of viral encephalitis have been reported, suggesting that the virus is neurotropic under unknown circumstances. This is well established for other coronaviruses. In order to understand why some patients develop such symptoms and others do not, we address herein the infectability of the central nervous system (CNS). Reports that the ACE2 receptor - critical for virus entry into lung cells - is found in different neurons support this expectation. We employed a human induced pluripotent stem cell (iPSC)derived BrainSphere model, which we used earlier for Zika, Dengue, HIV and John Cunningham virus infection studies. We detected the expression of the ACE2 receptor, but not TMPRSS2, in the model. Incubating the BrainSpheres for 6 hours with SARS-CoV-2 at a multiplicity of infection (MOI) of 0.1 led to infection of a fraction of neural cells with replication of the virus evident at $72 \mathrm{hpi}$. Virus particles were found in the neuronal cell body extending into apparent neurite structures. PCR measurements corroborated the replication of the virus, suggesting at least a tenfold increase in virus copies per total RNA. Leveraging state-of-the-art 3D organotypic cell culture, which has been shown to allow both virus infection and modeling of (developmental) neurotoxicity but is at the same time simple enough to be transferred and used in a BSL-3 environment, we demonstrate, for the first time, the potential critically important neurotropism of SARS-CoV-2.
\end{abstract}

\section{Introduction}

The question whether severe acute respiratory syndrome coronavirus 2 (SARS-CoV-2) infects brain cells and induces neuropathology or derails neural physiology is of critical importance for the understanding of this disease and its potential long-term sequelae. Several coronavirus species are neurotropic (Nath, 2020), but this has not been clearly established for SARSCoV-2. Initial reports from Wuhan, China, where the virus first emerged, indicate that $36 \%$ of COVID-19 patients have neurological symptoms (Mao et al., 2020), in the meantime confirmed by a European collective (Helms et al., 2020), and a case of acute hemorrhagic necrotizing encephalopathy, a rare encephalopathy that has been associated with other viral infections, has been reported in a COVID-19 patient (Poyiadji et al., 2020). One reason why some but not all patients show neurological manifestations could be that the blood-brain-barrier (BBB) normally hinders virus entry but is impaired in some by inflammatory conditions. The possible infection of brain endothelial cells was just reported (Paniz-Mondolfi et al., 2020).

The availability of experimental models to study SARS-CoV-2 is critical not only for drug development (Busquet et al., 2020) but also to understand environmental cofactors for public health measures. Viral infections are the prototypic species-specific dis-

\footnotetext{
\# contributed equally

Received June 11, 2020; Accepted June 26, 2020; Epub June 26, 2020; @ The Authors, 2020.

ALTEX 37(4), 665-671. doi:10.14573/altex.2006111

Correspondence: Lena Smirnova, PhD

Johns Hopkins Bloomberg School of Public Health

Department of Environmental Health \& Engineering

Center for Alternatives to Animal Testing (CAAT)

$615 \mathrm{~N}$ Wolfe St, W7032

Baltimore, MD, 21205, USA

(lena.smirnova@jhu.edu)
}

This is an Open Access article distributed under the terms of the Creative Commons Attribution 4.0 International license (http://creativecommons.org/licenses/by/4.0/), which permits unrestricted use, distribution and reproduction in any medium, provided the original work is appropriately cited. 
eases. There is no good small laboratory animal disease model for COVID-19. SARS-CoV-2 seems to be most closely related to several coronaviruses in Asian bats and reptiles, but these do not lend themselves easily as lab animals, and as far as we know they also do not display the human pathology (Zhou et al., 2020; Zhang et al., 2020). Recent reports on the infectability of macaques are of limited use with respect to throughput and species differences.

We reported in 2016 the first mass-produced standardized 3D human induced pluripotent stem cell (iPSC)-derived organotypic brain model (human mini-brains or BrainSpheres (Pamies et al., 2017; funded by NIH NCATS)), which consists of different types of neurons, astrocytes and oligodendrocytes. The promise of 3D organotypic models or microphysiological systems (MPS) for biomedical research has most recently been confirmed by our stakeholder workshop (Marx et al., 2020). While the battery of in vitro (developmental) neurotoxicological tests is broad, the BrainSphere model lends itself to these studies as it (i) is human-derived, a key prerequisite for studying human-specific viruses, (ii) has high cell density, a key characteristic for cell-to-cell infection of occasional neurotropic viruses, and (iii) allows central nervous system (CNS) functional assays. Our earlier studies with Zika and Dengue viruses in BrainSpheres demonstrated their applicability for studying neurotropic viruses (Abreu et al., 2018). In yet unpublished work, we infected the BrainSpheres with human immunodeficiency virus (HIV) and John Cunningham (JC) virus, where the latter could for the first time be cultured in a cell model (manuscript in preparation). Notably, there are limits as to the complexity of set-up that can be utilized for infectious disease research in BSL-3 facilities. Spheroids, pre-manufactured in regular cell culture laboratories, represent a compromise with respect to simplicity versus tissue architecture and functionality.

Here, the critical question of whether human brain cells can be infected with SARS-CoV-2 was addressed.

\section{Materials and methods}

\section{BrainSphere differentiation}

We previously developed a human 3D BrainSphere model derived from induced pluripotent stem cells (iPSC) with funding from NCATS, NIH (U18TR000547) (Pamies et al., 2017). Briefly, the differentiation protocol covers the stages from neural precursors to differentiated neurons (dopaminergic, glutamatergic, and GABAergic neurons, etc.) and glial cells (astrocytes and myelinating oligodendrocytes); BrainSpheres show electrophysiological activity. The BrainSpheres have been proven to be relevant to study key cellular processes involved in neurodevelopment and function including proliferation, differentiation, apoptosis, synaptogenesis, intracellular signaling, myelination, and neuronal network function (Pamies et al., 2017, 2018; Zhong et al., 2020).

BrainSpheres from the NIBSC-8 cell line (UK National Institute for Biological Standards and Control (NIBSC), kindly provided by Drs. Orla O'Shea and Elsa Abranches, UK stem cell bank) were differentiated following our in-house two-step protocol
(Pamies et al., 2017). The NIBSC-8 iPSC cell line is mycoplasma-free and of normal female karyotype, authenticated by short tandem repeat with $100 \%$ match to the donor MRC-9 cells (ATCC, CCL-212). Briefly, iPSCs were differentiated in a monolayer to neuroprogenitor cells (NPC) using serum-free, chemically defined neural induction medium (Gibco). NPC were expanded, and a single cell suspension was distributed into uncoated 6-well plates and cultured under constant gyratory shaking $(80 \mathrm{rpm}, 19 \mathrm{~mm}$ orbit) to form BrainSpheres. After $48 \mathrm{~h}$, differentiation was induced with serum-free, chemically defined BrainSphere differentiation medium (Neurobasal electro medium supplemented with B27-electro, glutamax, $10 \mathrm{ng} / \mathrm{mL}$ GDNF and $10 \mathrm{ng} / \mathrm{mL}$ BDNF). BrainSpheres were differentiated for eight weeks prior to infection. By this time, BrainSpheres consist of different types of neurons, astrocytes and oligodendrocytes (Pamies et al., 2017).

\section{Human gene quantitative reverse transcription polymerase chain reaction ( $R T-q P C R)$}

Expression of ACE2 and TMPRSS2 in BrainSpheres was assessed using TaqMan gene expression assay on an Applied Biosystems 7500 Fast Real-Time PCR machine. Briefly, total RNA was isolated with Zymo RNA isolation kit, $500 \mathrm{ng}$ RNA was reverse-transcribed with M-MLV-Reverse transcriptase (Promega) and amplified with ACE2 (Hs01085333_m1) and TMPRSS2 (Hs01122322_m1) TaqMan primers (ThermoFisher Scientific) following default Fast TaqMan cycling conditions. PCR experiments for human receptor expression were done in three independent experiments.

\section{SARS-CoV-2 infection of BrainSpheres}

SARS-CoV-2/Wuhan-1/2020 virus was provided to Dr Andrew Pekosz by the US Centers for Disease Control and Prevention (CDC). VeroE6 cells were used to grow the virus and determine the infectious virus titers as described earlier (Schaecher et al., 2007a,b). BrainSpheres were distributed into a 24-well plate at 15 spheres per well. BrainSpheres were then transferred to the BSL3 facility and infected with SARS-CoV-2/Wuhan-1/2020 (MOI 0.1). At $6 \mathrm{~h}$ post infection (hpi), BrainSpheres were washed by two-step wash and given fresh medium or lysed for RNA extraction. Supernatant samples were taken from the medium immediately after it was added to the BrainSpheres (6 hpi). BrainSpheres were incubated further until 72 hpi. At 72 hpi, supernatant samples were collected for quantification of virus RNA, and BrainSpheres were lysed for RNA extraction or fixed for immunohistochemistry. All infection experiments were conducted in two independent experiments in triplicates with appropriate MOCK controls included.

\section{Viral RNA RT-qPCR}

RNA from BrainSphere lysates was extracted using Zymo Quick RNA microPrep kit (Zymo Research) according to the manufacturer's protocol. On average, $600 \mathrm{ng}$ of total RNA was extracted per sample. A QIAamp Viral RNA mini kit (Qiagen) was used to isolate RNA from supernatants according to the manufacturer's protocol. cDNA was synthesized from the viral RNA using qScript cDNA SuperMix (Quantabio) following the manufacturer's protocol. The cDNA was quantified by RT-qP- 
CR on a StepOnePlus Real Time PCR system (Applied Biosystems) with TaqMan Fast Advanced Master Mix (Applied Biosystems). SARS-CoV-2 RNA was detected using premixed forward (5',-TTACAAACATTGGCCGCAAA-3') and reverse (5'-GCGCGACATTCCGAAGAA-3') primers and probe (5'-FAM-ACAATTTGCCCCCAGCGCTTCAG-BHQ1-3') designed by the CDC as part of the 2019-nCoV CDC Research Use Only (RUO) kit (Integrated DNA Technologies, Catalog \#10006713) to amplify a region of the SARS-CoV-2 nucleocapsid (N) gene. PCR conditions were as follows: $50^{\circ} \mathrm{C}$ for $2 \mathrm{~min}$, $95^{\circ} \mathrm{C}$ for $2 \mathrm{~min}$, followed by 45 cycles of $95^{\circ} \mathrm{C}$ for $3 \mathrm{~s}$ and $55^{\circ} \mathrm{C}$ for $30 \mathrm{~s}$. Serially diluted (10-fold) plasmid containing the complete SARS-CoV-2 N gene (Integrated DNA Technologies, Catalog \#10006625) was measured to generate a standard curve for quantification of viral RNA copies. The limit of detection for the assay was 1 x $10^{1}$ RNA copies. For cell lysates, viral copies were normalized to the human RNase $\mathrm{P}$ (RP) gene using premixed forward (5'-AGATTTGGACCTGCGAGCG-3') and reverse (5'-GAGCGGCTGTCTCCACAAGT-3') primers and probe (5'-FAM-TTCTGACCTGAAGGCTCTGCGCG-BHQ-1-3') included in the same 2019-nCoV CDC RUO kit.

\section{Immunohistochemistry}

At 72 hpi, BrainSpheres were fixed with 4\% paraformaldehyde and stained as described (Smirnova et al., 2015) with antiMAP2(a+b) antibody (Sigma), SARS-CoV-1 M-peptide rabbit antisera (McBride and Machamer, 2007) and mouse anti humanSARS-CoV/SARS-CoV-2 spike antibody (Sino Biological). Nuclei were stained with Hoechst 33342.

\section{Results}

\subsection{ACE2 but not TMPRSS2 is expressed in NPCs and mature BrainSpheres}

It has been shown that SARS-CoV-2 utilizes angiotensin-converting enzyme 2 (ACE2) receptors to enter cells and transmembrane serine protease 2 (TMPRSS2) for S protein priming (Hoffmann et al., 2020). Using quantitative RT-qPCR, we found the $A C E 2$ gene expressed in relatively low copy numbers in two different iPSC lines (NIBSC-8 (Fig. 1) and CRL-2097 (data not shown)). Notably, the receptor was already expressed in NPC. During differentiation, expression rates first dropped but then rebounded. Expression of the TMPRSS2 gene was below the detection limit in the BrainSpheres (data not shown).

\subsection{Immunohistochemical identification of SARS-CoV-2-infected neurons}

In order to analyze their infectability, BrainSpheres were fixed at 72 hpi and stained with fluorescent antibodies against neuronal marker MAP2, SARS-Cov-2 protein M, and spike protein (S). A small fraction of neural cells containing virus particles was detected by confocal microscopy (Fig. 2). Co-staining with neuronal marker MAP2 demonstrated the presence of the

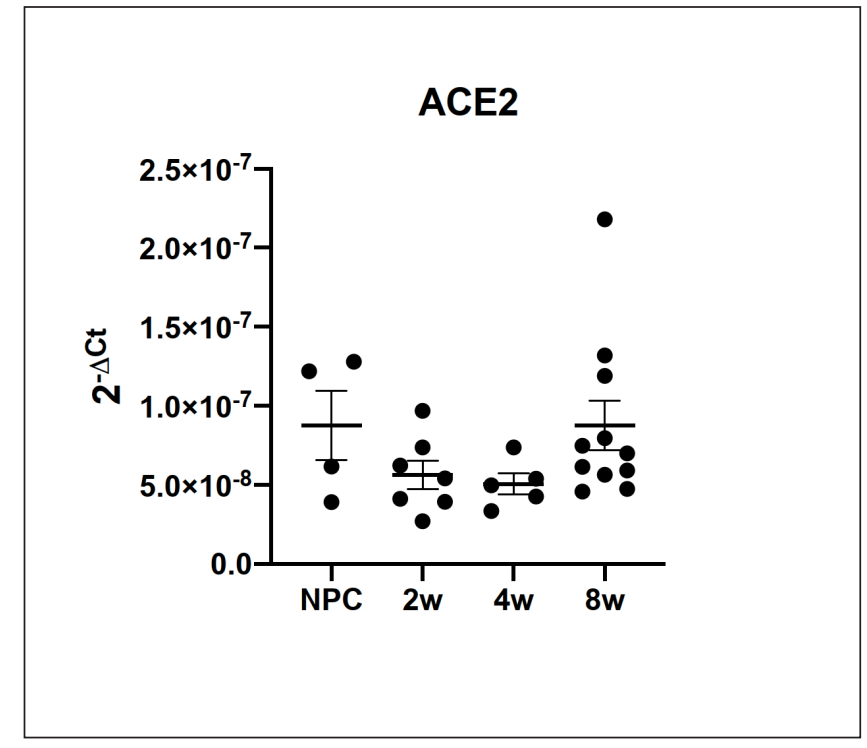

Fig. 1: Expression of angiotensin converting enzyme 2 receptor (ACE2) over time in human NIBSC-8 iPSC differentiated into neuroprogenitor cells (NPC) and BrainSpheres (for 2, 4 and 8 weeks)

Data shown combine three independent experiments with at least two biological replicates per condition; the 4-week timepoint was done in two independent experiments.

SARS-CoV-2 M protein in neuronal soma (Fig. 2C). In certain cells, the particles extended from the cell body into the neurites (Fig. 2B,D,E arrow heads). Similarly, neural cells were positive for SARS-CoV-2 spike protein (Fig. 2E,F). The large number of virus particles in the neurons is suggestive of active virus replication within the infected cells. In addition, some degree of cell bursting was observed in various cells (supplemental video $1^{1}$ ), presenting as non-cell-shaped clouds of virus, suggesting that the virus may initiate cell lysis.

\subsection{Increasing levels of viral RNA in SARS-CoV-2} infected BrainSpheres indicate viral genome replication In order to quantify cell-associated viral RNA, BrainSpheres were lysed at 6 and $72 \mathrm{hpi}$; supernatants were also collected at 6 and 72 hpi. We detected a baseline of cell-associated SARSCoV-2 genome copies in BrainSphere lysates at $6 \mathrm{hpi}$, which markedly increased by two log orders at 72 hpi (Fig. 3A). After extensive washing to remove the viral inoculum at $6 \mathrm{hpi}$, an unexpectedly high number of virus copies remained in the supernatant, which was greatly reduced at 72 hpi (Fig. 3B), possibly due to virus degradation in the supernatant over time and/or possible further penetration of virus from the supernatant into the spheres. This decrease of viral copies in the supernatant (35-fold) was significantly lower than the 107 -fold increase of viral copies in the BrainSphere lysate. This suggests that the increase in intracellular viral copies is due to virus replication within the cells rather

\footnotetext{
${ }^{1}$ https://share.getcloudapp.com/NQugZeb9
} 

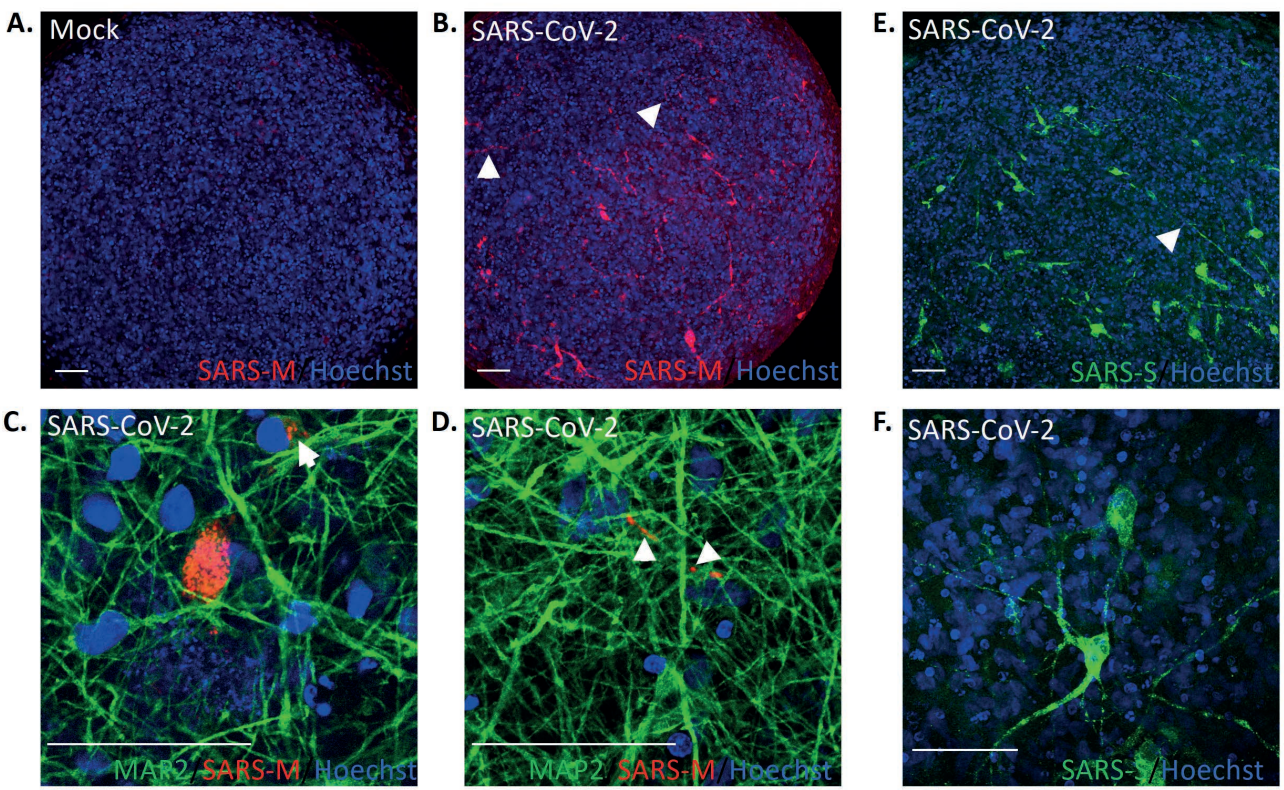

Fig. 2: A small fraction of neuronal cells in BrainSpheres contains virus particles at $72 \mathrm{hpi}$

BrainSpheres infected with SARS-CoV-2 at an MOI of 0.1 were analyzed at $72 \mathrm{hpi}$ for M protein (red) expression by immunofluorescence (A)-(D). Neuronal marker MAP2 (green) was used to stain the neurons. Arrowheads indicate colocalization and presence of the virus in neurites. (E) and (F) BrainSpheres stained for SARS-CoV-2 spike protein (green). Images representative of five BrainSpheres. Nuclei are stained with Hoechst 33342 (blue). Scale bar $50 \mu \mathrm{m}$.

than continued penetration of the residual virus from the supernatant over time. The detected viral copies in the supernatant after washing the BrainSpheres might be background from the remaining residual viral inoculum, similar to results reported in a MERS-CoV study (Chu et al., 2016).

In order to overcome the issue of high virus background in the supernatant, we made use of the fact that BrainSpheres are cultured in suspension, which allows more rigorous washing steps. In the repeat experiment, 6 hpi BrainSpheres were transferred into Eppendorf tubes, rigorously washed and plated into a new plate for incubation until $72 \mathrm{hpi}$. RNA was collected in triplicates from the supernatant and cell lysate after washing (6 hpi) and at 72 hpi from SARS-CoV-2 infected cells and corresponding MOCK control. In this experiment, the rigorous washing steps strongly decreased the number of viral particles in the supernatant at 6 hpi (Fig. 3D), but still resulted in a similar increase in virus copy numbers to about $10^{8}$ virus equivalents in cell lysates (Fig. 3C). This confirms that the intracellular increase in both experiments was due to virus replication and not continued penetration of the residual virus from the supernatant over time. Surprisingly, elimination of the high background at 6 hpi in the second experiment led even to an increase in viral copy numbers in the medium (Fig. 3D), suggesting virus shedding, which might be hidden in the first experiment due to the high level of residual viral inoculum. This confirmed the viral replication in the BrainSpheres and suggests a productive infection.

\section{Discussion}

The question whether SARS-CoV-2 infects brain cells and induces pathology or derails neural physiology is of critical importance for the understanding of this disease and its potential longterm sequelae. For this reason, we decided to report our initial findings with this short communication, at a moment when tens of thousands of patients are hospitalized. This might increase attention to neurological symptoms and add to the considerations on developing treatments.

The relatively frequent neurological symptoms in COVID-19 patients (Mao et al., 2020; Helms et al., 2020) prompted us to investigate whether SARS-CoV-2 can infect BrainSpheres directly. Notably, one of the key symptoms of COVID-19 appears to be a loss of the sense of smell, i.e., an olfactory dysfunction (Bagheri et al., 2020; Giacomelli et al., 2020), which was thought to indicate infection of the cranial nerve; however, most recently, it was suggested to be due to infection of non-neuronal cells in the olfactory system (Brann et al., 2020). More reports support the presence of virus in the CNS, e.g., the presence of viral particles in CSF biofluid (Zhou et al., 2020), which suggests a direct infection rather than a secondary immune response. Also, neural damage biomarkers were found in the plasma of patients with COVID-19 (Kanberg et al., 2020). The infectability of neural cells is not unexpected (Baig et al., 2020), as expression of the $A C E 2$ receptor gene, which was found to be critical for virus 


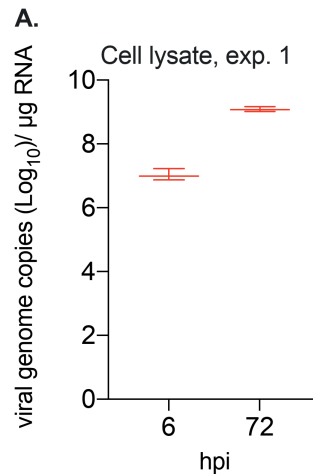

C.

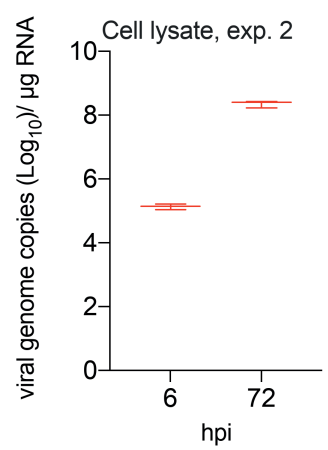

B.

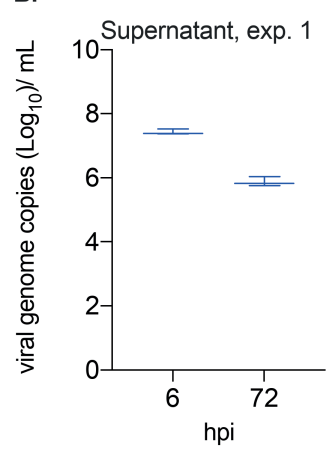

D.

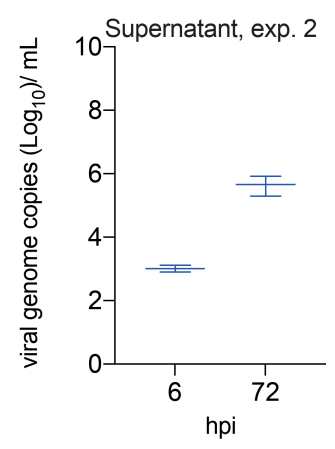

Fig. 3: SARS-CoV-2 replicates in infected human

\section{BrainSpheres}

(A) and (B) - experiment 1. (C) and (D) - experiment 2. Cell lysate associated SARS-CoV-2 genome levels presented as viral genome copies per $\mu \mathrm{g}$ total RNA (A and C) and SARS-CoV-2 genome copies per $\mathrm{mL}(600 \mu \mathrm{L}$ per well) in the culture supernatant (B and D) from 15 BrainSpheres (representing about 600 ng RNA) infected with SARS-CoV-2 at an MOI of 0.1 at 6 hpi and 72 hpi. Data are shown as box and whiskers plots displaying the data distribution through their quartiles at each time point from two experiments done in triplicate technical replicates $(n=3)$.

entry (Hoffmann et al., 2020), was detected in the BrainSpheres and has previously been shown by others in certain neurons (Xu et al., 2011; Chen et al., 2020). SARS-CoV-2 has been reported to use its spike $(S)$ proteins to facilitate viral entry through the ACE2 receptor and to use the serine protease TMPRSS2 for $\mathrm{S}$ protein priming (Hoffmann et al., 2020). We found no detectable expression of TMPRSS2 in the BrainSpheres, suggesting alternative processing.

The observation of bursting cells, irregular, non-cell-shaped clouds of virus (see supplemental video1), and the increase of viral particles in the supernatant over time indicate that infected cells shed virus particles, but it is unclear whether the infection is sustained by infecting other cells in our model. Future experiments with longer incubation times, longer hpi and/or higher MOI will be needed to answer these questions. It will be key to identify whether the infected cells represent a specific subpopulation of neurons and/or glia cells - for example, those express-

ing ACE2, as the rather low level of gene expression would suggest that only a subpopulation of neural cells expresses ACE2. Due to the low MOI used here, it could, however, also just be a probabilistic event where a few brain cells begin to be infected. The large number of virus particles in these cells in both experiments at 72 hpi suggests replication as corroborated by the PCR findings in the lysed BrainSpheres. Ongoing experiments aim to identify the neural cell type(s) that are infected.

These experiments require repetition, including varied initial number of virus (MOI), analysis of neural pathology and functionality, inclusion of different donors of iPSC of different genders, and extended time courses, etc. Virus replication, as evidenced by RT-qPCR and the accumulation of large numbers of virus particles in individual cells in this experiment can only occur when the virus infects living cells. Therefore, at least the fundamental possibility of CNS infection has been established in the experiments reported here. The aforementioned experiments will provide more insight into the mechanistic processes underlying CNS infection. It is important to note that no major damage to the BrainSpheres was noted. However, this might change when incubation periods are prolonged and higher virus titers are used or when functional assays address more subtle changes.

The majority of patients might, however, be well protected by a functioning BBB, and this should be included in future research. A shortcoming of the BrainSphere model is the absence of immune cells, which in the brain are the microglia, which do not derive from neural precursor cells but from the mesoderm germ layer and invade the developing brain from the blood. Our studies with Dengue, Zika (Abreu et al., 2018), HIV and JC virus (manuscript in preparation) already investigated the effects of adding microglia to BrainSpheres, which resulted in cytokine release and neuronal damage. It will be important to follow up on this initial SARS-CoV-2 research with immunocompetent BrainSpheres.

The BrainSpheres represent a model of the developing brain and have been used to demonstrate the developmental neurotoxicity of pesticides (Pamies et al., 2018) and drugs (Zhong et al., 2020). It will be of critical importance to show whether neurodevelopment is affected by SARS-CoV-2 infection. Importantly, transplacental transmission of SARS-CoV-2 has been reported in a neonate born to a mother infected in the last trimester (Vivanti et al., 2020), which caused placental inflammation and neurological manifestations in the neonate consistent with those observed in infected adults. As the BBB is not functional in the most critical early months of brain development (Serlin et al., 2015), there is limited protection against exogenous infections. Clinical evidence cannot yet be expected, however, as most children at critical phases of embryo and fetal development are yet to be born, and more subtle neurodevelopmental disorders often take time to manifest and diagnose after birth. It will be important to study whether brain infection occurs also in otherwise asymptomatic patients, especially children, with possible long-term consequences.

This study provides evidence that SARS-CoV-2 can infect neural cells, likely contributing to neurological outcomes and possibly to neurodevelopmental disorders. This means we have to face yet another potentially devastating pathomechanism of 
COVID-19. The observed infectability and replication warrant further research to help treat this disease and protect especially vulnerable populations, such as those exposed to neurotoxicants, pregnant women, and possibly those with pre-existing neurological conditions. In the future, it is of fundamental importance to understand whether SARS-CoV-2 impacts not only the mature but also the developing brain. This model uniquely lends itself to addressing these questions. Overall, this study stresses the versatility of microphysiological systems (MPS) (Marx et al., 2016, 2020) as enabling technologies beyond the replacement of animal tests.

\section{References}

Abreu, C. M., Gama, L., Krasemann, S. et al. (2018). Microglia increase inflammatory responses in iPSC-derived human BrainSpheres. Front Microbiol 9, 2766. doi:10.3389/ fmicb.2018.02766

Bagheri, S. H., Asghari, A., Farhadi, M. et al. (2020). Coincidence of COVID-19 epidemic and olfactory dysfunction outbreak in Iran. Med J Islam Repub Iran 34, 446-452.

Baig, A. M., Khaleeq, A., Ali, U. et al. (2020). Evidence of the COVID-19 virus targeting the CNS: Tissue distribution, host-virus interaction, and proposed neurotropic mechanisms. ACS Chem Neurosci 11, 995-998. doi:10.1021/acschemneuro.0c00122

Brann, D. H., Tsukahara, T., Weinreb, C. et al. (2020). Non-neuronal expression of SARS-CoV-2 entry genes in the olfactory system suggests mechanisms underlying COVID-19-associated anosmia. Sci Adv 6, eabc5801. doi:10.1126/sciadv.abc5801

Busquet, F., Hartung, T., Rovida, C. et al. (2020). Harnessing the power of novel animal-free test methods for the development of COVID-19 drugs and vaccines. Arch Toxicol 94, 22632272. doi:10.1007/s00204-020-02787-2

Chen, R., Wang, K., Yu, J. et al. (2020). The spatial and cell-type distribution of SARS-CoV-2 receptor ACE2 in human and mouse brain. Bioxiv, preprint. doi:10.1101/2020.04.07.030650

Chu, H., Zhou, J., Wong, B. H.-Y. et al. (2016). Middle East respiratory syndrome coronavirus efficiently infects human primary $\mathrm{T}$ lymphocytes and activates the extrinsic and intrinsic apoptosis pathways. J Infect Dis 213, 904-914. doi:10.1093/ infdis/jiv380

Giacomelli, A., Pezzati, L., Conti, F. et al. (2020). Self-reported olfactory and taste disorders in SARS-CoV-2 patients: A cross-sectional study. Clin Infect Dis 71, 889-890. doi:10.1093/ cid/ciaa330

Helms, J., Kremer, S., Merdji, H. et al. (2020). Neurologic features in severe SARS-CoV-2 infection. N Engl J Med 382, 2268-2270. doi:10.1056/NEJMc2008597

Hoffmann, M., Kleine-Weber, H., Schroeder, S. et al. (2020). SARS-CoV-2 cell entry depends on ACE2 and TMPRSS2 and is blocked by a clinically proven protease inhibitor. Cell 181, 271-280.e8. doi:10.1016/j.cell.2020.02.052

Kanberg, N., Ashton, N. J., Andersson, L.-M. et al. (2020). Neurochemical evidence of astrocytic and neuronal injury commonly found in COVID-19. Neurology, 10.1212/WNL. 0000000000010111. doi:10.1212/WNL.0000000000010111

Mao, L., Jin, H., Wang, M. et al. (2020). Neurologic manifesta- tions of hospitalized patients with coronavirus disease 2019 in Wuhan, China. JAMA Neurol 77, 683-690. doi:10.1001/jama neurol.2020.1127

Marx, U., Andersson, T. B., Bahinski, A. et al. (2016). Biology-inspired microphysiological system approaches to solve the prediction dilemma of substance testing using animals. ALTEX 33, 272-321. doi:10.14573/altex.1603161

Marx, U., Akabane, T., Andersson, T. B. et al. (2020). Biology-inspired microphysiological systems to advance medicines for patient benefit and animal welfare. ALTEX 37, 364-394. doi:10.14573/altex.2001241

McBride, C. E., Li, J. and Machamer, C. E. (2007). The cytoplasmic tail of the severe acute respiratory syndrome coronavirus spike protein contains a novel endoplasmic reticulum retrieval signal that binds COPI and promotes interaction with membrane protein. J Virol 81, 2418-2428. doi:10.1128/JVI.02146-06

Nath, A. (2020). Neurologic complications of coronavirus infections. Neurology 94, 809-810. doi:10.1212/WNL. 0000000000009455

Pamies, D., Barreras, P., Block, K. et al. (2017). A human brain microphysiological system derived from iPSC to study central nervous system toxicity and disease. ALTEX 34, 362-376. doi:10.14573/altex.1609122

Pamies, D., Block, K., Lau, P. et al. (2018). Rotenone exerts developmental neurotoxicity in a human brain spheroid model. Toxicol Appl Pharmacol 354, 101-114. doi:10.1016/j.taap.2018.02.003

Paniz-Mondolfi, A., Bryce, C., Grimes, Z. et al. (2020). Central nervous system involvement by severe acute respiratory syndrome coronavirus-2 (SARS-CoV-2). J Med Virol 92, 699702. doi:10.1002/jmv.25915

Poyiadji, N., Shahin, G., Noujaim, D. et al. (2020). COVID-19-associated acute hemorrhagic necrotizing encephalopathy: Imaging features. Radiology 296, E119-E120. doi:10.1148/ radiol.2020201187

Schaecher, S. R., Touchette, E., Schriewer, J. et al. (2007a). Severe acute respiratory syndrome coronavirus gene 7 products contribute to virus-induced apoptosis. J Virol 81, 1105411068. doi:10.1128/JVI.01266-07

Schaecher, S. R., Mackenzie, J. M. and Pekosz, A. (2007b). The ORF7b protein of severe acute respiratory syndrome coronavirus (SARS-CoV) is expressed in virus-infected cells and incorporated into SARS-CoV particles. J Virol 81, 718-731. doi:10.1128/JVI.01691-06

Serlin, Y., Shelef, I., Knyazer, B. et al. (2015). Anatomy and physiology of the blood-brain barrier. Semin Cell Dev Biol 38, 2-6. doi:10.1016/j.semcdb.2015.01.002

Smirnova, L., Harris, G., Delp, J. et al. (2015). A LUHMES 3D dopaminergic neuronal model for neurotoxicity testing allowing long-term exposure and cellular resilience analysis. Arch Toxicol 90, 2725-2743. doi:10.1007/s00204-015-1637-z

Vivanti, A., Vauloup-Fellous, C., Prevot, S. et al. (2020). Transplacental transmission of SARS-CoV-2 infection. Nat Commun 11, 3572. doi:10.1038/s41467-020-17436-6

Xu, P., Sriramula, S. and Lazartigues, E. (2011). ACE2/ANG(1-7)/Mas pathway in the brain: The axis of good. Am J Physiol Regul Integr Comp Physiol 300, R804-817. doi:10.1152/ ajpregu.00222.2010 
Zhang, T., Wu, Q. and Zhang, Z. (2020). Probable pangolin origin of SARS-CoV-2 associated with the COVID-19 outbreak. Curr Biol 30, 1346-1351.e2. doi:10.1016/j.cub.2020.03.022

Zhong, X., Harris, G., Smirnova, L. et al. (2020). Antidepressant paroxetine exerts developmental neurotoxicity in an iPSC derived 3D human brain model. Front Cell Neurosci 14, 25. doi:10.3389/fncel.2020.00025

Zhou, H., Chen, X., Hu, T. et al. (2020). A novel bat coronavirus closely related to SARS-CoV-2 contains natural insertions at the S1/S2 cleavage site of the spike protein. Curr Biol 30, 2196-2203.e3. doi:10.1016/j.cub.2020.05.023

Zhou, L., Zhang, M., Wang, J. et al. (2020). Sars-Cov-2: Underestimated damage to nervous system. Travel Med Infect Dis 36, 101642. doi:10.1016/j.tmaid.2020.101642

\section{Conflict of interest}

$\mathrm{TH}$ and $\mathrm{HH}$ are named inventors on a patent by Johns Hopkins University on the production of mini-brains (also called BrainSpheres), which is licensed to AxoSim, New Orleans, LA, USA. $\mathrm{TH}, \mathrm{LS}$ and HH consult AxoSim, and TH is a shareholder.

\section{Acknowledgment}

This work was financially supported by NIH R21 135527 , R01AI52688 and through the generosity of the collective community of donors to the Johns Hopkins University School of Medicine for COVID-19 research. Expert editing of the article by Mike Hughes is gratefully appreciated.

\title{
Non-aversive Mouse Handling Techniques: A Survey on the Acceptance and Concerns of the Animal Facility and Scientific Staff
}

\author{
Katharina Hohlbaum ${ }^{1}$, Melanie Humpenöder ${ }^{1}$, Stefan Nagel-Riedasch ${ }^{2}$, Kristina Ullmann ${ }^{2,3}$ \\ and Christa Thöne-Reineke1 \\ ${ }^{1}$ Institute of Animal Welfare, Animal Behavior, and Laboratory Animal Science, Department of Veterinary Medicine, Freie Universität Berlin, Berlin, \\ Germany; ${ }^{2}$ Research Facilities for Experimental Medicine (FEM), Charité - Universitätsmedizin Berlin, Berlin, Germany; ${ }^{3}$ Charité 3R, Charité - \\ Universitätsmedizin Berlin, Berlin, Germany
}

Routine laboratory procedures such as handling can cause stress and anxiety in animals, which in turn compromise animal welfare and are confounding factors in animal experimentation (Balcombe et al., 2004; Bailey, 2017). This underlines the need to develop and implement appropriate refinement strategies for animal handling techniques to minimize stress of animals when interacting with humans.

Laboratory mice are usually picked up by their tails. However, a decade ago, this traditional handling technique was refined by Hurst and West (2010) by introducing tunnel and cup handling. If a mouse is picked up using a tunnel or by cupping it on the open hand, it will experience less anxiety and stress when compared to a mouse that is gripped and lifted at the base of its tail (Hurst and West, 2010). The benefits for both animal welfare and quali- ty of research data associated with tunnel and cup handling appear to have recently convinced many stakeholders: More and more working groups have adopted tunnel and cup handling in their laboratory routines, and even entire institutions are considering to replace tail handling with the refined mouse handling techniques.

To introduce non-aversive mouse handling at the Research Facilities for Experimental Medicine (FEM), Charité - Universitätsmedizin Berlin, three courses on non-aversive mouse handling techniques were offered in November and December 2019. Each course had the same content and comprised a 40-min lecture on tunnel and cup handling in mice and approximately 20 min discussion. All staff having contact with laboratory mice, i.e., animal caretakers, technical assistants, scientists, and veterinarians, had the opportunity to attend one of the courses and
Received May 12, 2020;

(c) The Authors, 2020.

ALTEX 37(4), 671-674. doi:10.14573/altex.2005121

Correspondence: Katharina Hohlbaum, PhD

Freie Universität Berlin, Department of Veterinary Medicine

Institute of Animal Welfare, Animal Behavior, and Laboratory Animal Science

Königsweg 67 (Haus 21)

14163 Berlin, Germany

(katharina.hohlbaum@fu-berlin.de)
This is an Open Access article distributed under the terms of the Creative Commons Attribution 4.0 International license (http://creativecommons.org/licenses/by/4.0/) which permits unrestricted use, distribution and reproduction in any medium, provided the original work is appropriately cited. 